\title{
COMPLETE SETS OF LOGICAL FUNCTIONS
}

\author{
BY \\ WILLIAM WERNICK
}

A. In the two-valued calculus of sentences we denote variables by " $p$," " $q$," and their truth values by " 1, " " 0. " A truth function of two variables will be a function which assumes the value 1 , or 0 , depending on the truth values of the variables $p, q$, in its argument. Since each variable may assume independently the value 1,0 , there are $2^{2}$ possible arguments for each function. A particular function is completely determined if we assign to each of its 4 possible arguments a value which may be independently 1 , or 0 , so there are altogether $2^{2^{2}}$, i.e., 16 possible functions in this calculus. Some of these functions are familiar to us by name: conjunction, implication, negation, etc. When we wish to speak of all 16 functions collectively, we shall call the totality " $C$."

Sheffer has shown $\left({ }^{1}\right)$ that we may take one of these as undefined and define formally in terms of this one all the other functions of the calculus. $\mathrm{He}$ also pointed out that this function, which he called "stroke," could be given a dual interpretation, that is, there are two functions in $C$, mutually dual, which, alone, can define all the other 15 functions in $C$. Whitehead and Russell use the pair of undefined functions negation and disjunction to generate this calculus; Hilbert and Ackerman show $\left({ }^{2}\right)$ that this pair will suffice, as well as the pairs negation and conjunction, or negation and implication. They show also that the pair negation and equivalence is insufficient to generate the calculus, being incapable, in particular, of defining conjunction.

B. If we take as undefined the set of functions $\{\alpha, \beta, \cdots, \delta\}$ we shall look for such sets which have the following properties:

1. All the 16 functions of $C$ may be formally $\left({ }^{3}\right)$ defined in terms of functions of this set. (Sufficiency.)

2. No function of this set may be defined in terms of some or all the remaining functions of the set. (Non-redundancy.)

If a set of undefined functions is both sufficient and non-redundant, it will be called "complete." Thus the set negation and disjunction is a complete

Presented to the Society, February 24, 1940; received by the editors March 11, 1940 and, in revised form, December 10,1940. This paper is the substance of the author's doctoral thesis at New York University, February, 1941. The author wishes to extend thanks to Dr. J. C. C. McKinsey for his interest in this investigation.

(') H. M. Sheffer, these Transactions, vol. 14 (1913), pp. 481-488.

(2) Hilbert and Ackerman, Grundz üge der theoretischen Logik, 2d edition, 1938, p. $8 \mathrm{ff}$.

(3) A definition will be called "formal" if the definiens is simply an expression built up by the use of sentential connectives (our functions) and sentential variables " $p$," " $q$." All other definitions are non-formal. 
set. This paper will enumerate all complete sets and show that they may contain one, two, or three functions, but not more. Post points out $\left({ }^{4}\right)$ the possibility of generalizing the Principia development by using other primitive (i.e., generating) functions than negation and disjunction, but does not discuss complete sets of such functions $\left({ }^{5}\right)$.

C. We may start by listing the 16 functions thus:

TABLE I

\begin{tabular}{|ll|lllllllllllllllll|}
\hline \hline$p$ & $q$ & $a$ & $b$ & $c$ & $d$ & $e$ & $f$ & $g$ & $h$ & $a^{\prime}$ & $b^{\prime}$ & $c^{\prime}$ & $d^{\prime}$ & $e^{\prime}$ & $f^{\prime}$ & $g^{\prime}$ & $h^{\prime}$ \\
\hline 1 & 1 & 0 & 1 & 0 & 0 & 0 & 1 & 1 & 1 & 1 & 0 & 1 & 1 & 1 & 0 & 0 & 0 \\
1 & 0 & 0 & 0 & 1 & 0 & 0 & 1 & 0 & 0 & 1 & 1 & 0 & 1 & 1 & 0 & 1 & 1 \\
0 & 1 & 0 & 0 & 0 & 1 & 0 & 0 & 1 & 0 & 1 & 1 & 1 & 0 & 1 & 1 & 0 & 1 \\
0 & 0 & 0 & 0 & 0 & 0 & 1 & 0 & 0 & 1 & 1 & 1 & 1 & 1 & 0 & 1 & 1 & 0 \\
\hline
\end{tabular}

We shall take our arguments in what follows so that the truth values of $p, q$, will always be in the order 1,$1 ; 1,0 ; 0,1 ; 0,0$. The names given to these functions are immaterial, and the use of " $a^{\prime}$," " $b^{\prime}$," etc., instead of " $i$,", " $j$," etc., has only mnemonic significance. We may note some remarks on this table:

1. The function $b(p, q)$, defined above, may be recognized as the truth function "logical product," $p \cdot q$, which is true only when $p$ and $q$ are both true. Interpretations of all the 16 functions, in the more familiar notation of symbolic logic, are given here, with the emphasis on familiarity, and not on economy of symbols:

TABLE II

\begin{tabular}{|l|l|l|l|}
\hline \hline$a: p \cdot \sim p$ & $e: \sim p \cdot \sim q$ & $a^{\prime}: p \vee \sim p$ & $e^{\prime}: p \vee q$ \\
$b: p \cdot q$ & $f: p$ & $b^{\prime}: \sim(p \cdot q)$ & $f^{\prime}: \sim p$ \\
$c: \sim(p \supset q)$ & $g: q$ & $c^{\prime}: p \supseteq q$ & $g^{\prime}: \sim q$ \\
$d: \sim(q \supset p)$ & $h: p \equiv q$ & $d^{\prime}: q \supset p$ & $h^{\prime}: \sim(p \equiv q)$ \\
\hline
\end{tabular}

2. The function $f(p, q)$ need never be an undefined function, since it is immediately definable with no additional machinery. The function $f(p, q)$ is exactly $p$, being true when and only when $p$ is true, as may be seen from Table I. Likewise $g(p, q)=q$. This means in any truth function involving $p$ or $q$, we may replace $p$ by $f(p, q)$, or $q$ by $g(p, q)\left(^{6}\right)$.

3. We introduce here the notion of symmetric functions. The function $\alpha$

(4) E. L. Post, Introduction to a general theory of elementary propositions, American Journal of Mathematics, vol. 43 (1921), pp. 163-185.

(5) His concept of a complete system should not be confused with our concept of a complete set of functions. He calls a system of truth tables "complete" if it contains all possible truth tables of the logic. Thus, the listing of all 16 functions of $C$ in Table I below would form a complete system in Post's notation. He uses the notion of redundancy of a set of generating functions only implicitly (when he speaks of the "order" of a system).

(6) We use "or" throughout this paper to mean either alternative or both. 
will be called symmetric to the function $\beta$, if the following four equations all hold:

$$
\begin{array}{ll}
\alpha(1,1)=\beta(1,1), & \alpha(0,1)=\beta(1,0), \\
\alpha(1,0)=\beta(0,1), & \alpha(0,0)=\beta(0,0) .
\end{array}
$$

Note that if $\alpha(1,0)=\alpha(0,1)$, then $\alpha$ is self-symmetric, i.e., the value is independent of the order of the arguments. If, in Table II, we replace " $p$ " by " $q$," and " $q$ " by " $p$ " in the definition of any particular function, we obtain the definition of its symmetric function. If there resulted no change (due to the commutative nature of the connectives $\cdot, \vee$, and $\equiv)$, then that function was self-symmetric. The reader can easily verify the list of self-symmetric functions: $a, b, e, h, a^{\prime}, b^{\prime}, e^{\prime}, h^{\prime}$; and the others symmetric in pairs: $c S d$; $f S g ; c^{\prime} S d^{\prime}$; and $f^{\prime} S g^{\prime}$.

4. Since we are dealing only with functions of two arguments, any function of $p$ alone will be considered as a function of both $p$ and $q$ which is independent of $q$; e.g., $f^{\prime}(p, q)$ is the function " $p$ is false," and is independent of $q$. Similarly for a function of $q$ alone.

5. The fact that our functions are defined for arguments of the form $p, q$, as $p, q$ go through their ordered changes, means that it is unnecessary to consider functions of $q, p$. For example, $c^{\prime}(p, q)$ is our definition for " $p$ implies $q$," and we may not use $c^{\prime}(q, p)$ to mean " $q$ implies $p$," but must use some function of $p, q$, since our definitions are set up on the agreement that the first place in the table is the value of the function for $p=1$ and $q=1$, the second place is the value for $p=1$ and $q=0$, etc. Introduction of arguments of the form $q, p$ would disrupt our definitions. They are, moreover, superfluous, since any function of $q, p$ has an equivalent function of $p, q$.

In view of the theorem proved in $\S \mathrm{H} 1$ we remark, that so far as completeness is concerned, the functions $d, d^{\prime}, g, g^{\prime}$ are equivalent to $c, c^{\prime}, f, f^{\prime}$, respectively. We shall omit, from now on, further unnecessary reference to $d, d^{\prime}, g, g^{\prime}$ when they are to be undefined functions. They continue to play their roles as functions of $C$ to be defined by others.

D. We introduce now an abbreviated notation which will greatly reduce the amount and complexity of the material to follow. Consider the function of functions $e\left[h(p, q), e^{\prime}(p, q)\right]$. As $p, q$ go through their ordered changes $(1,1 ; 1,0 ; 0,1 ; 0,0)$ we get the values $0,0,0,0$.

But this set of values is the same set we would have obtained if we had let $p, q$ go through their ordered changes for the function $a(p, q)$ (see Table I). This establishes the identity $e\left[h(p, q), e^{\prime}(p, q)\right]=a(p, q)$ for all values of $p, q$. This identity will now be written simply as $e\left(h, e^{\prime}\right)=a$.

E. We consider, from now on, only functions of the form $\alpha(\beta, \gamma)$, where $\alpha, \beta, \gamma$ are any of the 16 functions in $C$. The functions $f, g$ play a special role here, as noted in $\S \mathrm{C}$, since $\alpha(f, g)=\alpha(p, q)$. While we do not define $\alpha(p, p)$, we have defined $\alpha(f, f)$, which is equivalent to it. 
Consider the sets of functions defined thus:

$\{\alpha\}_{0}: f, g$;

$\{\alpha\}_{1}: \alpha(f, f) ; \quad \alpha(f, g) ; \quad \alpha(g, f) ; \quad \alpha(g, g) ;$

$\{\alpha\}_{i}$ : all functions in $\left\{\{\alpha\}_{i-1}\right\}$ with $f$ or $g$ or both replaced by any functions in $\left\{\{\alpha\}_{k}\right\}, k<i-1$.

Clearly, $\{\alpha\}_{1}$ will contain the function $\alpha(p, q)$, since this is equal to $\alpha(f, g)$, and may, from considerations in $\S \mathrm{C}$, contain other functions of $p, q$; e.g., if $\alpha$ is the function $e$ then $f^{\prime}=e(f, f)$. Also, $\{\alpha\}_{2}$ may contain functions not in $\{\alpha\}_{1}$; and $\{\alpha\}_{3}$ may contain functions not in $\{\alpha\}_{2}$ or in $\{\alpha\}_{1}$. (It is understood that all functions that occur in the above constructions can be reduced to, and are replaced by, the equivalent single functions of $C$.) This accretion of new functions must soon stop, since there are only 16 different functions altogether. The totality of all (reduced) functions of $p, q$, in all the sets $\left\{\{\alpha\}_{i}\right\}$, where the $i$ is sufficiently large to insure that no new functions can be introduced by increasing $i$, will be called the field of the function $\alpha$, indicated by " $F(\alpha)$;" thus, from the beginning of this paragraph we have: $f^{\prime}$ is in $F(e)$. The process of enumerating a field is always finite, and there are immediately evident to an investigator special theorems for each particular function that reduce the work of enumeration. Since we are concerned only with the totality of functions in $F(\alpha)$, in extension and without repetitions, we can immediately discard repetitions of functions in $C$ which we have already found in $\left\{\{\alpha\}_{i-1}\right\}$ before investigating $\left.\{\alpha\}_{i}{ }^{7}\right)$. The problem: "What functions of $C$ are in $F(\alpha)$ ?" is seen to be equivalent to the problem: "What functions in the calculus of sentences can be defined, if we take a particular function $\alpha$ as undefined?" ( $\left.{ }^{8}\right)$.

F. If there are two undefined functions $\alpha, \beta$, we have analogously the sets of functions

$\{\alpha, \beta\}_{0}: f, g$;

$\{\alpha, \beta\}_{1}:\{\alpha\}_{1}+\{\beta\}_{1}$

$\{\alpha, \beta\}_{i}$ : all functions in $\left\{\{\alpha, \beta\}_{i-1}\right\}$ with $f$ or $g$ or both replaced by any function

$$
\text { in }\left\{\{\alpha, \beta\}_{k}\right\}, k<i-1 \text {. }
$$

(7) E. Żyliński, Some remarks concerning the theory of deduction, Fundamenta Mathematicae, vol. 7 (1925), pp. 203-209 also discusses, with different terminology, the notion of a field. $\mathrm{He}$ asks, in effect, for the field for each function and indicates the results given in Table III below, but does not discuss fields of two or more functions.

${ }^{8}$ ) Since $f$, and $g$, can be defined without the aid of any function, they properly belong in every field. These two functions can be formally defined in terms of 14 of our 16 functions, and cannot be formally defined in terms of $a$, or $a^{\prime}$; nevertheless, we place $f$ and $g$ in $F(a)$, and in $F\left(a^{\prime}\right)$, since we have, for example, the definitions: $a(f, g)=a=a(p, q) ; f(f, g)=p=f(p, q)$; $g(f, g)=q=g(p, q)$; with only the function $a$ as an undefined function. 
Note that $\{\alpha, \beta\}_{2}$ certainly includes $\{\alpha\}_{2}+\{\beta\}_{2}$ but may easily include more functions. The totality of functions (reduced and without repetitions) in all the sets $\left\{\{\alpha, \beta\}_{i}\right\}$ will be called the field of the functions $\alpha, \beta$, indicated by " $F(\alpha, \beta)$." As before, the process of enumerating a field is always finite and becomes quickly simplified in any particular case by the application of simple particularized theorems which apply to each pair. As before, the problem: "What functions are in $F(\alpha, \beta)$ ?" is equivalent to the problem: "What functions in the calculus of sentences can be defined in terms of only two given undefined functions?"

There are corresponding definitions for sets and fields of three undefined functions $\alpha, \beta, \gamma$; of four undefined functions, etc. We may point out here that Post (loc. cit.) discusses the totality of functions formally definable in terms of a set of $u$ arbitrary functions $\left\{f_{1}, \cdots, f_{u}\right\}$, where $f_{i}$ is a function of $m_{i}$ arguments. This totality of functions is called $F$, and corresponds to our notion of a field. He does not concern himself with redundancy among the $\left\{f_{i}\right\}$.

Since we are concerned with sufficient sets of functions, we shall try to find functions whose field is the entire set of 16, i.e., whose field is $C$. Also, since we are looking for non-redundant sets of functions, we shall discard a set $\{\alpha, \beta, \gamma, \delta, \cdots\}$ if, for example, $\alpha$ is in $F(\beta, \gamma, \delta, \cdots)$.

G. We now prove that the functions $e$ and $b^{\prime}$ are the only single functions that form complete sets $\left({ }^{9}\right)$. Obviously, the set $\{e\}$ is non-redundant, as is the set $\left\{b^{\prime}\right\}$. If we are seeking a single function $\alpha$, so that $F(\alpha)=C$, it must satisfy the condition that $\alpha(1,1)=0$, and $\alpha(0,0)=1\left({ }^{10}\right)$. For, if $\alpha(1,1)=1$ then any function of $\alpha$ would have as its first pair of arguments the truth values 1,1 , and hence, for those arguments, would have the value 1 , whereas half the functions in $C$ have the value 0 for the first.pair of arguments. Similar reasoning assures that $\alpha(0,0)$ must equal 1 . Looking through Table I for functions of the form $0,-,-, 1$; we find only the functions $e, b^{\prime}, f^{\prime}, g^{\prime}$. A function of one variable cannot define a function really dependent on two variables, so that our function $\alpha$ which is to define all the functions in $C$ may not be independent of one of its arguments. But we quickly establish the fact that $f^{\prime}$ is independent of $q$, and $g^{\prime}$ is independent of $p$ (since $f^{\prime}(1,1)=f^{\prime}(1,0)$, and $f^{\prime}(0,1)=f^{\prime}(0,0)$; and similarly for $\left.g^{\prime}\right)$. Hence $e$ and $b^{\prime}$ are the only possible functions.

Since Sheffer (loc. cit.) has already shown that these two functions are sufficient, this completes the proof that the only complete sets consisting of single functions are $\{e\}$, and $\left\{b^{\prime}\right\}$.

$\mathrm{H}$. If we look for complete sets consisting of two functions, the realm of

(9) These two functions are Sheffer's stroke function and its dual. He states (loc. cit.), in effect, that these two sets are complete. Żyliński (loc. cit.) states, in effect, but does not prove, that these are the only complete sets of single functions. The proof is given here in full because it is typical.

$\left({ }^{10}\right)$ This is essentially the property of negation. 
investigation broadens considerably. There seem to be $C_{16,2}$, i.e., 120 possible pairs of functions to investigate, but some obvious considerations will quickly reduce this number. Any pair containing the functions $e$, or $b^{\prime}$ is, by $\S \mathrm{G}$, immediately redundant, since all functions are in $F(e)$, and in $F\left(b^{\prime}\right)$. Also, any pair containing $f$ or $g$ is redundant, since, from the definition of a field, these functions are in every field. Not all of the remaining $C_{12,2}$, i.e., 66 pairs of functions are essentially different, since, in view of the theorem on symmetric functions mentioned in $\S \mathrm{C}$ above, any function pair containing $d, d^{\prime}$, or $g^{\prime}$ is equivalent, as far as definability is concerned, to the same pair with $d, d^{\prime}$, or $g^{\prime}$ replaced by $c, c^{\prime}$, or $f^{\prime}$, respectively. This leaves only 36 pairs of functions to investigate. To find the redundant functions, we determine first the fields of single functions as per Table III( $\left.{ }^{11}\right)$.

TABLE III

\begin{tabular}{l|l|}
\hline \hline$F(a)=a, f, g$ & $F\left(a^{\prime}\right)=a^{\prime}, f, g$ \\
$F(b)=b, f, g$ & $F\left(b^{\prime}\right)=C$ \\
$F(c)=a, b, c, d, f, g$ & $F\left(c^{\prime}\right)=f, g, a^{\prime}, c^{\prime}, d^{\prime}, e^{\prime}$ \\
$F(d)=a, b, c, d, f, g$ & $F\left(d^{\prime}\right)=f, g, a^{\prime}, c^{\prime}, d^{\prime}, e^{\prime}$ \\
$F(e)=C$ & $F\left(e^{\prime}\right)=f, g, e^{\prime}$ \\
$F(f)=f, g$ & $F\left(f^{\prime}\right)=f, g, f^{\prime}, g^{\prime}$ \\
$F(g)=f, g$ & $F\left(g^{\prime}\right)=f, g, f^{\prime}, g^{\prime}$ \\
$F(h)=f, g, h, a^{\prime}$ & $F\left(h^{\prime}\right)=a, f, g, h^{\prime}$ \\
\hline
\end{tabular}

We note some remarks on Table III :

1. Symmetry. The identity of the fields for certain functions was predicted by a theorem mentioned earlier: "If $\alpha$ is symmetric to $\beta$, then $F(\alpha)=F(\beta)$," which we now prove.

If $\alpha$ is symmetric to $\beta$, and $\epsilon$ is in $F(\alpha)$, then there must exist functions $\gamma, \delta$, in $F(\alpha)$ so that $\epsilon=\alpha(\gamma, \delta)$. From the definition of symmetry, we must also have $\epsilon=\beta(\delta, \gamma)$, and $\epsilon$, therefore, will be in $F(\beta)$ if $\gamma, \delta$ are in $F(\beta)$. Applying to $\gamma, \delta$ the same argument we have just applied to $\epsilon$, we form a recursion in a very few steps to $\{\alpha\}_{0}$, and $\{\beta\}_{0}$. But these are, by definition, identical, consisting of the functions $f$, and $g$; hence the theorem.

2. Transitivity. The reader can verify the rather obvious theorem: "If $\alpha$ is in $F(\beta)$, and $\beta$ is in $F(\gamma)$, then $\alpha$ is in $F(\gamma)$." In terms of definability, this theorem states that if $\beta$ can define $\alpha$, and $\gamma$ can define $\beta$, then $\gamma$ can define $\alpha$. As a corollary to this theorem on transitivity, which seems intuitively evident, we can derive the theorem given above under symmetry; for, if $\alpha$ is symmetric to $\beta$ then clearly $\alpha$ is in $F(\beta)$.

3. Duality. The function $\alpha$ is called the dual of the function $\beta$, notation $\alpha=\bar{\beta}$, if, for every $p$ and $q$, we have $\alpha(p, q)=\sim \beta(\sim p, \sim q)$. We use " $\sim$ " in

(11) For purposes of completeness, we indicate the fields for each of the 16 functions in $C$, though we shall not need all these fields for the remainder of this paper. The results in this table also appear in Żyliński's paper (loc. cit.). 
its usual sense, so that $\sim 1=0$, and $\sim 0=1$. We state the useful and simple theorem: "If $\alpha=\bar{\beta}$, then $\beta=\bar{\alpha}$," whose proof is left to the reader.

We list here the relations of duality that exist among the functions in $C$ :

TABLE IV

\begin{tabular}{|c|l|l|l|l|}
\hline \hline $\begin{array}{l}a=\overline{a^{\prime}} \\
b=\overline{e^{\prime}}\end{array}$ & $\begin{array}{l}c=\overline{d^{\prime}} \\
d=\overline{c^{\prime}}\end{array}$ & $\begin{array}{l}e=\overline{b^{\prime}} \\
f=\bar{f}\end{array}$ & $\begin{array}{l}g=\bar{g} \\
h=\overline{h^{\prime}}\end{array}$ & $\begin{array}{l}f^{\prime}=\overline{f^{\prime}} \\
g^{\prime}=\overline{g^{\prime}}\end{array}$ \\
\hline
\end{tabular}

This table may be verified in the following way, suggested by Żyliński (loc. cit.). In Table I, replace " 0 " by " 1 ," and " 1 " by " 0 " everywhere; then the matrix definition under each function designation will be that of the dual of that function. This amounts to interchanging " 0 " and " 1 " in the definitions, and then writing the columns upside down. The equation we have given to define duality is, of course, equivalent to this transformation.

From Tables III and IV we could easily verify the theorem: "If $\alpha$ is in $F(\beta)$, then $\bar{\alpha}$ is in $F(\bar{\beta})$," the usefulness of which will be more apparent if we state it in the form: "If $\beta=\bar{\alpha}$, and $F(\alpha)=\gamma_{1}, \cdots, \gamma_{n}$, then $F(\beta) \overline{\gamma_{1}}=, \cdots, \overline{\gamma_{n}}$." To prove it directly, we first prove that if $\alpha=\beta(\gamma, \delta)$ then $\bar{\alpha}=\bar{\beta}(\bar{\gamma}, \bar{\delta})$. Our hypothesis, written out in full is: "For every $p$, and $q$, we have the identity $\alpha(p, q)=\beta(\gamma(p, q), \delta(p, q))$." Replace " $p$ " by " $\sim p$," " $q$ " by " $\sim q$," and preface both sides of the equation by " $\sim$." This gives us

$$
\sim \alpha(\sim p, \sim q)=\sim \beta(\gamma(\sim p, \sim q), \delta(\sim p, \sim q)) .
$$

From the definition of dual functions, we have $\bar{\alpha}(p, q)=\sim \alpha(\sim p, \sim q)$, or, by prefacing both sides of this equation by " $\sim$," and dropping " $\sim$," $\sim \bar{\alpha}(p, q)=\alpha(\sim p, \sim q)$. Applying these equations to (1), we have

$$
\bar{\alpha}(p, q)=\sim \beta(\sim \bar{\gamma}(p, q), \sim \bar{\delta}(p, q)),
$$

which can be abbreviated to

$$
\bar{\alpha}=\sim \beta(\sim \bar{\gamma}, \sim \bar{\delta}) .
$$

But, again, from the definition of duality, we have $\bar{\beta}(p, q)=\sim \beta(\sim p, \sim q)$, and by replacing " $p$ " by " $\bar{\gamma}$," and " $q$ " by " $\bar{\delta}$," we have $\bar{\beta}(\bar{\gamma}, \bar{\delta})=\sim \beta(\sim \bar{\gamma}, \sim \bar{\delta})$; therefore, finally $\bar{\alpha}=\bar{\beta}(\bar{\gamma}, \bar{\delta})$.

To say that $\alpha$ is in $F(\beta)$ is to say that there is an equation $\alpha=\beta(\gamma, \delta)$, where $\gamma, \delta$ are in $F(\beta)$. But if there is such an equation, we have just shown that there is an equally valid equation $\bar{\alpha}=\bar{\beta}(\bar{\gamma}, \bar{\delta})$; that is, $\bar{\alpha}$ is in $F(\bar{\beta})$, provided only that $\bar{\gamma}, \bar{\delta}$ are in $F(\bar{\beta})$. We have, now, a simple recursion, applying to $\gamma, \delta$ the same arguments as to $\alpha$, and leading to $\{\beta\}_{0}$ and $\{\bar{\beta}\}_{0}$, that is, our theorem depends on: "If the functions in $\{\beta\}_{0}$ are in $F\{\beta\}$, then the functions in $\{\bar{\beta}\}_{0}$ are in $F(\bar{\beta})$;" which is, of course, a true theorem, since $\{\epsilon\}_{0}=f, g$, for any $\epsilon$. Hence our main theorem.

These properties of duality may be used to check our results later on, since 
all definitions may be dualized and yield equally valid definitions. (If, e.g., $\{\alpha, \beta\}$ is a complete set, so must $\{\bar{\alpha}, \bar{\beta}\}$ be.)

4. Identity of fields. Since $F(c)=F(d) ; F(e)=F\left(b^{\prime}\right) ; F(f)=F(g) ; F\left(c^{\prime}\right)$ $=F\left(d^{\prime}\right) ; F\left(f^{\prime}\right)=F\left(g^{\prime}\right)$; there are just 11 different fields generated by a single function. Post (loc. cit.) has investigated systems of functions definable by functions of more than two variables. He states, in effect, that there are just 66 different fields which can be generated by functions of fewer than four arguments, and there are 8 infinite families which can be generated by functions of four or more arguments. He does not discuss, however, such concepts as duality, symmetry, and transitivity.

We shall not verify the enumeration of all fields in Table III, but shall indicate the method by which this may be done.

There are two steps in the verification of $F(\alpha)$. First we must prove that the functions listed as belonging to $F(\alpha)$ are actually in $F(\alpha)$, which could be done immediately by giving the definitions in terms of $\alpha$, or of functions defined in terms of $\alpha$, etc. (using transitivity). Second, we must show that no other function belongs in that field, i.e., that the field is closed. We may use special theorems for each function, but we can always use this straightforward method of proving closure: If $\delta$ is in $F(\alpha)$, then there must be a definition $\delta=\alpha(\beta, \gamma)$, where $\beta, \gamma$ are in $F(\alpha)$. If we have already shown that there are, say, six functions in $F(\alpha)$, we can substitute for $\beta$, or $\gamma$, any of these six functions, giving, in this case, 36 possible pairs of argument functions. Closure is proved if the values resulting from these 36 arguments are among the six functions already shown to be in $F(\alpha)$. It is never necessary to investigate the result of every substitution, since special theorems give the results for many classes of substitutions.

The verification of most of the fields is straightforward, but we verify $F(c)$ in detail to illustrate another typical method, $F(c)=a, b, c, d, f, g ; a=c(f, f)$; $b=c(f, c) ; d=c(g, f)$. If $\alpha$ is any of the above six functions in $F(c)$, then $\alpha(0,0)=0$ (see Tahle I). Therefore (see $\S G$ ) any function of these six functions in $F(c)$ will reduce, for $p=0, q=0$, to the value 0 . Hence $F(c)$ consists of at most the eight functions in $C$ for which the argument 0,0 gives the value 0 . To prove closure, we must now show that $e^{\prime}$ and $h^{\prime}$, the only other functions which have this property, are not in $F(c)$. From the definition of $c, c(0, x)=0$, for all $x$, and $c(1, y)=1$ only when $y=0$. Therefore, $c(\alpha, \beta)$ can have the value 1 at most as many times as $\alpha$ has the value 1 , and then at the same places. If $e^{\prime}$ is in $F(c)$ then there must exist functions $\alpha, \beta$, in $F(c)$ such that $e^{\prime}=c(\alpha, \beta)$, where, furthermore, $\alpha$ is of the form $1,1,1, x ; \alpha$, in turn, requires in its definition another function with the same form, etc. Therefore, there must be in $\{c\}_{0}$ a function of the form $1,1,1, x$; which is not the case. Therefore, $e^{\prime}$ is not in $F(c)$. Similarly, $h^{\prime}$ in $F(c)$ requires eventually that $\{c\}_{0}$ contain a function of the form $x, 1,1, y$; which it does not contain. Therefore, $h^{\prime}$ is not in $F(c)$. This concludes the proof that $F(c)$ is closed as given. 
From Table III we may now easily select the redundant pairs of functions. We indicate them, 67 in all, in the following table $\left({ }^{12}\right)$ :

TABLE V



The 54 pairs of redundant functions are not written down, since they are obvious, but they, and the 13 pairs directly above, comprise all the redundant function pairs, i.e., pairs of which one function (not either one) may be taken as undefined, and the other will be immediately definable. Of these first 13 pairs, only 6 are essentially different and must be subtracted from the 36 pairs mentioned at the beginning of $\S \mathrm{H}$ (e.g., the pairs $a, c ; a, d$ are not essentially different, since $c S d$, and $F(a, c)=F(a, d))$.

I. We now have left to consider, from the total of 120 pairs of functions indicated in $\$ \mathrm{H}$, only 30 essentially different pairs which are not redundant, but whose sufficiency is still undecided. We determine, by the method shown in $\S \mathrm{F}$, the fields for each of these 30 pairs, and find that there are exactly 9 pairs which form sufficient sets. Since these are selected from non-redundant pairs, these 9 sets are, in fact, complete sets of two functions, and are listed below. This listing answers completely the question: What pairs of functions may be used to define the calculus of sentences?

TABLE VI

\begin{tabular}{|l|l|l|}
\hline \hline$a, c^{\prime}$ & $c, a^{\prime}$ & $c^{\prime}, f^{\prime}$ \\
$b, f^{\prime}$ & $c, c^{\prime}$ & $c^{\prime}, h^{\prime}$ \\
$c, h$ & $c, f^{\prime}$ & $e^{\prime}, f^{\prime}$ \\
\hline
\end{tabular}

This table may be represented by the following diagram:

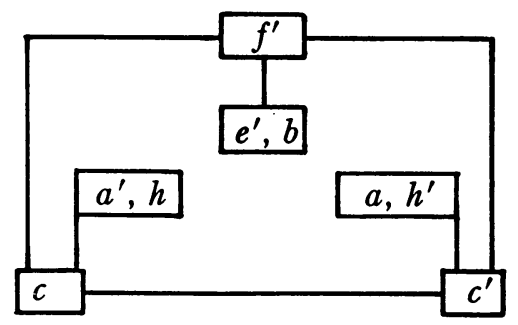

(12) We shall leave out, in the text and tables from now on the braces around functions even though we mean them to be generating sets, whether complete or not. 
Any function in one box may be paired with a function in another box to which it is joined by a line, to form a complete two-function set. The preceding diagram can be translated in an obvious way (see Table II) to yield the following diagram, of some interest in the calculus of sentences, since it gives all essentially different pairs of functions which can define this calculus:

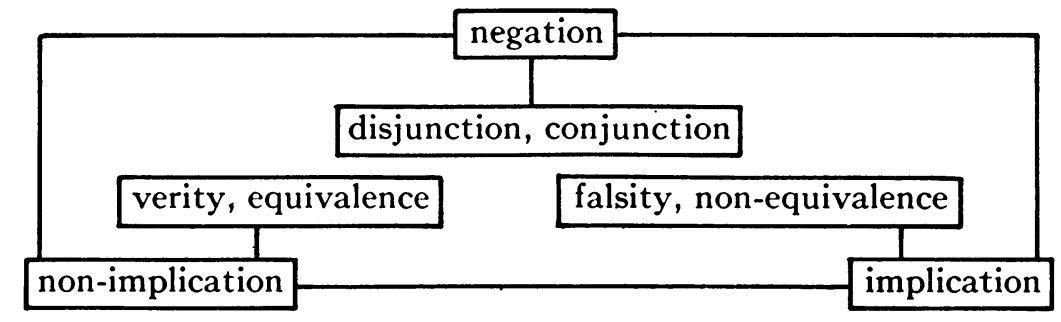

It is possible to show the sufficiency of these pairs without actually enumerating the fields for each pair, by applying the theorem stated in the remark on transitivity, following Table III. Since we have shown that

$$
F(e)=F\left(b^{\prime}\right)=C,
$$

as soon as we can show that $e$ or $b^{\prime}$ is in $F(\alpha, \beta)$, it follows immediately that

$$
F(\alpha, \beta)=C \text {. }
$$

We do this now for all the pairs in Table VI. In most cases, a single definition will suffice, but we need at most two defined functions. The definitions given here are not the only possible definitions for the function actually defined, and we define $e$ or $b^{\prime}$, depending on which comes first to hand, or seems easier.

TABLE VII

\begin{tabular}{|l|l|}
\hline \hline$a, c^{\prime}: f^{\prime}=c^{\prime}(f, a), b^{\prime}=c^{\prime}\left(c^{\prime}, f^{\prime}\right)$ & $c^{\prime}, f^{\prime}: b^{\prime}=c^{\prime}\left(g, f^{\prime}\right)$ \\
$c, a^{\prime}: f^{\prime}=c\left(a^{\prime}, f\right), e=c\left(f^{\prime}, g\right)$ & $c^{\prime}, h^{\prime}: b^{\prime}=h^{\prime}\left(f, c^{\prime}\right)$ \\
$b, f^{\prime}: b^{\prime}=f^{\prime}(b, f)$ & $e^{\prime}, f^{\prime}: e=f^{\prime}\left(e^{\prime}, f\right)$ \\
$c, h: b^{\prime}=h(f, c)$ & \\
$c, c^{\prime}: e=c\left(c^{\prime}, g\right)$ & \\
$c, f^{\prime}: e=c\left(f^{\prime}, g\right)$ & \\
\hline
\end{tabular}

If Tables VI and VII had included all complete pairs (i.e., those given, and those obtainable from them by replacing $c, c^{\prime}, f^{\prime}$ by $d, d^{\prime}, g^{\prime}$, respectively), we could have used a duality theorem to check our tables; e.g., from the complete set $c, h$ and its definition of $b^{\prime}$, we could obtain the dual (complete) set $d^{\prime}, h^{\prime}$ and its definition of $e$.

$\mathrm{J}$. There still remain, of the 30 non-redundant pairs mentioned in $\S \mathrm{I}$, a total of 21 pairs of functions which form non-redundant but insufficient sets. We list them in Table VIII below: 
TABle VIII

\begin{tabular}{|l|l|l|l|l|l|l|}
\hline \hline$a, b$ & $a, e^{\prime}$ & $b, a^{\prime}$ & $b, h^{\prime}$ & $h, c^{\prime}$ & $h, h^{\prime}$ & $a^{\prime}, h^{\prime}$ \\
$a, h$ & $a, f^{\prime}$ & $b, c^{\prime}$ & $c, e^{\prime}$ & $h, e^{\prime}$ & $a^{\prime}, e^{\prime}$ & $e^{\prime}, h^{\prime}$ \\
$a, a^{\prime}$ & $b, h$ & $b, e^{\prime}$ & $c, h^{\prime}$ & $h, f^{\prime}$ & $a^{\prime}, f^{\prime}$ & $f^{\prime}, h^{\prime}$ \\
\hline
\end{tabular}

The assertion here that these pairs are insufficient can only be substantiated if we list and verify the fields for each of the 21 pairs in Table VIII, which we shall do in more detail than we did for single functions in Table III.

We shall list the functions in $F(\alpha, \beta)$ thus: $F(\alpha, \beta)=\gamma_{1}, \cdots, \gamma_{p} ; \delta_{1}, \cdots, \delta_{q}$; $\epsilon_{1}, \cdots, \epsilon_{r}$, where $\gamma_{i}$ is in $F(\alpha), \delta_{i}$ is in $F(\alpha)+F(\beta)$ but not in $F(\alpha)$, and $\epsilon_{i}$ is in $F(\alpha, \beta)$ but not in $F(\alpha)+F(\beta)$. We shall give definitions only for the functions $\epsilon_{i}\left({ }^{13}\right)$, since definitions for $\gamma_{i}$ and $\delta_{i}$ have already been indicated in $\S \mathrm{H}$. Proofs of closure to show that no more functions than those listed belong to $F(\alpha, \beta)$ are given after the listing of the fields in Table IX below:

\section{TABLE IX}

\begin{tabular}{|c|c|c|}
\hline 1. $F(a, b)$ & $=a, f, g ; b$ & \\
\hline 2. $F(a, h)$ & $=a, f, g ; h, a^{\prime} ; f^{\prime}, g^{\prime}, h^{\prime}$ & $f^{\prime}=h(f, a) ; g^{\prime}=h(g, a) ; h^{\prime}=h(h, a)$ \\
\hline 3. $F\left(a, a^{\prime}\right)$ & $=a, f, g ; a^{\prime}$ & \\
\hline 4. $F\left(a, e^{\prime}\right)$ & $=a, f, g ; e^{\prime}$ & \\
\hline 5. $F\left(a, f^{\prime}\right)$ & $=a, f, g ; f^{\prime}, g^{\prime} ; a^{\prime}$ & $a^{\prime}=f^{\prime}(a, f)$ \\
\hline 6. $F(b, h)$ & $=b, f, g ; h, a^{\prime} ; c^{\prime}, d^{\prime}, e^{\prime}$ & $c^{\prime}=h(f, b) ; d^{\prime}=h(g, b) ; e^{\prime}=h(b, h)$ \\
\hline 7. $F\left(b, a^{\prime}\right)$ & $=b, f, g ; a^{\prime}$ & \\
\hline 8. $F\left(b, c^{\prime}\right)$ & $=b, f, g ; a^{\prime}, c^{\prime}, d^{\prime}, e^{\prime} ; h$ & $h=b\left(c^{\prime}, d^{\prime}\right)$ \\
\hline 9. $F\left(b, e^{\prime}\right)$ & $=b, f, g ; e^{\prime}$ & \\
\hline 10. $F\left(b, h^{\prime}\right)$ & $=b, f, g ; a, h^{\prime} ; c, d, e^{\prime}$ & $c=h^{\prime}(f, b) ; d=h^{\prime}(g, b) ; e^{\prime}=h^{\prime}(f, d)$ \\
\hline 11. $F\left(c, e^{\prime}\right)$ & $=a, b, c, d, f, g ; e^{\prime} ; h^{\prime}$ & $h^{\prime}=c\left(e^{\prime}, b\right)$ \\
\hline 12. $F\left(c, h^{\prime}\right)$ & $=a, b, c, d, f, g ; h^{\prime} ; e^{\prime}$ & $e^{\prime}=h^{\prime}(g, c)$ \\
\hline 13. $F\left(h, c^{\prime}\right)$ & $=f, g, h, a^{\prime} ; c^{\prime}, d^{\prime}, e^{\prime} ; b$ & $b=h\left(g, d^{\prime}\right)$ \\
\hline 14. $F\left(h, e^{\prime}\right)$ & $=f, g, h, a^{\prime} ; e^{\prime} ; b, c^{\prime}, d^{\prime}$ & $b=h\left(h, e^{\prime}\right) ; c^{\prime}=h\left(g, e^{\prime}\right) ; d^{\prime}=h(g, b)$ \\
\hline 15. $F\left(h, f^{\prime}\right)$ & $=f, g, h, a^{\prime} ; f^{\prime}, g^{\prime} ; a, h^{\prime}$ & $a=f^{\prime}\left(a^{\prime}, f\right) ; h^{\prime}=f^{\prime}(h, f)$ \\
\hline 16. $F\left(h, h^{\prime}\right)$ & $=f, g, h, a^{\prime} ; a, h^{\prime} ; f^{\prime}, g^{\prime}$ & $f^{\prime}=h^{\prime}(g, h) ; g^{\prime}=h^{\prime}(f, h)$ \\
\hline 17. $F\left(a^{\prime}, e^{\prime}\right)$ & $=f, g, a^{\prime} ; e^{\prime}$ & \\
\hline 18. $F\left(a^{\prime}, f^{\prime}\right)$ & $=f, g, a^{\prime} ; f^{\prime}, g^{\prime} ; a$ & $a=f^{\prime}\left(a^{\prime}, f\right)$ \\
\hline 19. $F\left(a^{\prime}, h^{\prime}\right)$ & $=f, g, a^{\prime} ; a, h^{\prime} ; h, f^{\prime}, g^{\prime}$ & $h=h^{\prime}\left(a^{\prime}, h^{\prime}\right) ; f^{\prime}=h^{\prime}\left(a^{\prime}, f\right) ; g^{\prime}=h^{\prime}\left(a^{\prime}, g\right)$ \\
\hline 20. $F\left(e^{\prime}, h^{\prime}\right)$ & $=f, g, e^{\prime} ; a, h^{\prime} ; b, c, d$ & $b=h^{\prime}\left(e^{\prime}, h^{\prime}\right) ; c=h^{\prime}(f, b) ; d=h^{\prime}(g, b)$ \\
\hline 21. $F\left(f^{\prime}, h^{\prime}\right)$ & $=f, g, f^{\prime}, g^{\prime} ; a, h^{\prime} ; h, a^{\prime}$ & $h=h^{\prime}\left(f^{\prime}, g\right) ; a^{\prime}=f^{\prime}(a, f)$ \\
\hline
\end{tabular}

We may prove closure for several of these fields at once. Notice that many of the fields are identical and often consist of eight functions. Consider these:

${ }^{(13)}$ We list the complete fields and definitions for all functions $\epsilon_{i}$, including even the defined functions $d, d^{\prime}, g^{\prime}$, which, we know, must appear in any field in which their respective symmetrics $c, c^{\prime}, f^{\prime}$ appear. 


$$
\begin{aligned}
& F_{1}: \quad a, b, c, d, f, g, e^{\prime}, h^{\prime}, \\
& F_{2}: \quad b, f, g, h, a^{\prime}, c^{\prime}, d^{\prime}, e^{\prime}, \\
& F_{3}: \quad a, f, g, h, a^{\prime}, f^{\prime}, g^{\prime}, h^{\prime} .
\end{aligned}
$$

If we examine the definitions of these functions in Table I, we may note:

(i) $F_{1}$ consists of all functions $\{\alpha\}$ in $C$ for which $\alpha(0,0)=0$;

(ii) $F_{2}$ consists of all functions $\{\alpha\}$ in $C$ for which $\alpha(1,1)=1$;

(iii) $F_{3}$ consists of all functions $\{\alpha\}$ in $C$ for which the sum of the four constants defining $\alpha$ is even, i.e., $F_{3}$ consists of all even functions.

We shall prove a general theorem: "If $\alpha, \beta, \cdots, \delta$, are all in $F_{i}(i=1,2,3)$, then every function in $F(\alpha, \beta, \cdots, \delta)$ is in $F_{i}$."

$F_{1}$ : From the remark at the beginning of $\S \mathrm{G}$, we know that if $\alpha(0,0)=0$, and if $\epsilon$ is in $F(\alpha)$, then we can never have $\epsilon(0,0)=1$. That is, if $\alpha$ is in $F_{1}$, then every function in $F(\alpha)$ is also in $F_{1}$. This argument holds as well if there are two functions to generate the field: if $\alpha$ and $\beta$ are in $F_{1}$, then any function defined in terms of $\alpha$ and $\beta$ (i.e., any function in $F(\alpha, \beta)$ ) must always have for $p=0, q=0$, the value 0 , i.e., must be in $F_{1}$. The extension to several functions, $\alpha, \beta, \cdots, \delta$, all of which are in $F_{1}$ is immediate.

$F_{2}$ : Referring to the same remark as above, for functions $\alpha$ for which $\alpha(1,1)=1$, we derive in the same way an analogous conclusion: if, for $p=1$, $q=1$, the functions $\alpha, \beta, \cdots, \delta$, take the value 1 , then every function in $F(\alpha, \beta, \cdots, \delta)$ must also have this property. But the condition of this statement is precisely that $\alpha, \beta, \cdots, \delta$ all be in $F_{2}$, and the conclusion that every function in $F(\alpha, \beta, \cdots, \delta)$ is also in $F_{2}$.

$F_{3}$ : Consider functions of the form $\alpha(\beta, \gamma)$, where $\alpha, \beta, \gamma$ may be replaced by any function in $F_{3}$. We must show that the result is always a function in $F_{3}$. Consider replacements of $\alpha$ by functions in $F_{3}$. We shall use known properties of these functions in the form of theorem-equations without proof (which would follow immediately from their definitions). We have for every $\beta, \gamma$, the following:

$$
\begin{array}{ll}
a(\beta, \gamma)=a, & a^{\prime}(\beta, \gamma)=a^{\prime}, \\
f(\beta, \gamma)=\beta, & f^{\prime}(\beta, \gamma)=\beta^{\prime}, \\
g(\beta, \gamma)=\gamma, & g^{\prime}(\beta, \gamma)=\gamma^{\prime} .
\end{array}
$$

If $\beta, \gamma$ are in $F_{3}$, clearly $\beta^{\prime}$ and $\gamma^{\prime}$ are also in $F_{3}$, and the results of all the above replacements are also in $F_{3}$; so we need now investigate the cases $h(\beta, \gamma)$, and $h^{\prime}(\beta, \gamma)$. But again, from the definitions we have

$$
h^{\prime}(\beta, \gamma)=(h(\beta, \gamma))^{\prime},
$$

so we need now investigate only $h(\beta, \gamma)$. Note that this involves more than $F(h)$, since $\beta$ or $\gamma$ need not be in $F(h)$, but must be in $F_{3}$, which contains more than $F(h)$. 
We have also the theorem-equation

$$
h\left(\beta, \gamma^{\prime}\right)=(h(\beta, \gamma))^{\prime},
$$

which makes it unnecessary to investigate primed arguments; so we need to investigate only replacements of $\beta, \gamma$ by $a, f, g, h$. And now, finally, these last theorems:

$$
h(\gamma, a)=h(a, \gamma)=\gamma^{\prime} ; \quad h(\beta, \beta)=a^{\prime} ;
$$

make it necessary to test only the three possibilities $h(f, g), h(f, h), h(g, h)$. But all these are, of course, in $F(h)$ which is contained in $F_{3}$, their primes are likewise in $F_{3}$, and the last part of our general theorem is thus established.

This last part may be stated: "An odd function can never be in a field generated only by even functions," and can be used for checking many fields without proving closure. For example: $b$ is not in $F\left(h, f^{\prime}\right)$, since $h, f^{\prime}$ are even and can only generate an even field, whereas $b$ is odd( $\left.{ }^{14}\right)$.

We may obtain some immediate corollaries:

COROLlaRy 1. Every complete set must contain at least one odd function.

Corollary 2. A necessary condition that the set $\alpha, \beta, \cdots, \delta$ be complete is that not all the functions $\alpha, \beta, \cdots, \delta$ be in some $F_{i}$.

Corollary 3. Consider the sets of functions:

$$
F_{4}: \quad a, f, g, a^{\prime}, f^{\prime}, g^{\prime} ; \quad F_{5}: a, a^{\prime} ;
$$

which are subsets of $F_{3}$. We have, from the last part of the proof above, the corollary that the general theorem includes the cases $i=4,5$.

We derive now some important consequences of this general theorem. Among the 21 pairs in Table VIII we notice many pairs which have both their members in some $F_{i}$, and hence come within the scope of this theorem. In particular, the four pairs $b, h^{\prime} ; c, e^{\prime} ; c, h^{\prime} ; e^{\prime}, h^{\prime}$ are wholly in $F_{1}$ and hence, by the theorem, their fields are at most $F_{1}$. But we have already shown that their fields are at least $F_{1}$; therefore, their fields are exactly $F_{1}$. Similarly, there are four pairs which are wholly in $F_{2}: b, h ; b, c^{\prime} ; h, c^{\prime} ; h, e^{\prime}$; to which we may apply exactly the same method. We have shown that their fields are at least $F_{2}$, and the theorem states that their fields are at most $F_{2}$; therefore, their fields are exactly $F_{2}$. The theorem is still applicable to the five function pairs wholly in $F_{3} ; a, h ; h, f^{\prime} ; h, h^{\prime} ; a^{\prime}, h^{\prime} ; f^{\prime}, h^{\prime} ;$ whose fields are therefore proved to be closed.

We have, with the general theorem, proved closure for 13 of our 21 fields. Corollary 3 may be used to prove closure for three more fields. In particular,

${ }^{(14)}$ That is, the pair of functions negation and equivalence are incapable of defining conjunction, as stated by Hilbert and Ackerman (loc. cit.). 
the two pairs $a, f^{\prime}$ and $a^{\prime}, f^{\prime}$ are in $F_{4}$; therefore, by the usual argument, their fields are exactly $F_{4}$. Also, the pair $a, a^{\prime}$ is in $F_{5}$; therefore, its field is exactly $F_{5}$.

This still leaves the five pairs $a, b ; a^{\prime}, b ; a, e^{\prime} ; a^{\prime}, e^{\prime} ; b, e^{\prime} ;$ for whose fields we must prove closure. We know, for any $\alpha, \beta$, that

$$
a(\alpha, \beta)=a ; \quad a^{\prime}(\alpha, \beta)=a^{\prime} .
$$

Also, from the definitions of $b$, and $e^{\prime}$, which are self-symmetric, that $b(a, \alpha)=a$, $b\left(a^{\prime}, \alpha\right)=\alpha, e^{\prime}(a, \alpha)=\alpha$, and $e^{\prime}\left(a^{\prime}, \alpha\right)=a^{\prime}$. (This follows, since $b(x, y)=1$, only when $x=y=1 ; e^{\prime}(x, y)=1$ when $x$ or $y=1 ; a$ is always 0 ; and $a^{\prime}$ is always 1.) Obvious applications of these theorem-equations will prove closure for $F(a, b)$, $F\left(a^{\prime}, b\right), F\left(a, e^{\prime}\right)$, and $F\left(a^{\prime}, e^{\prime}\right)$.

Now we have only $F\left(b, e^{\prime}\right)$ to investigate. But, if $\alpha$ is either of these functions, by their definitions $\alpha(1,1)=1$ and $\alpha(0,0)=0$; that is, $F\left(b, e^{\prime}\right)$ is at most the intersection of $F_{1}$ and $F_{2}$. But these functions are exactly $b, f, g, e^{\prime}$, which are already known to be in $F\left(b, e^{\prime}\right)$. Therefore, $F\left(b, e^{\prime}\right)$ is closed.

This completes the verification of the fields for the 21 pairs of non-redundant, insufficient function pairs listed in Table VIII $\left({ }^{15}\right)$.

$\mathrm{K}$. If we consider, now, complete sets of three undefined functions, the field of investigation does not broaden immediately, as one might think. The condition of non-redundancy forces us to reject a triad $\alpha, \beta, \gamma$, which contains a pair which forms either a redundant or a complete set; for in the first case, the triad would reduce, insofar as defining power is concerned, to a pair; and in the second case, the triad would be redundant, though no pair in it need be, that is, the admissible triads will be only those such that the three pairs which it is possible to select from them be all non-redundant and insufficient, that is, be all in Table VIII.

If we examine Table VIII in this way, we find a total of only ten nonredundant triads (which establishes, incidentally, that of the $C_{16,3}$, that is, 560 possible triads, only ten are non-redundant) and determine, as in $\S \S \mathrm{E}$ and $\mathrm{F}$, the fields for each of these ten triads. Of the ten triads, six were found to be sufficient, that is, we discovered six complete sets of three undefined functions, as follows:

TABLE X

\begin{tabular}{|l|l|l|}
\hline \hline $\begin{array}{l}a, b, h \\
a, h, e^{\prime}\end{array}$ & $\begin{array}{l}b, h, h^{\prime} \\
b, a^{\prime}, h^{\prime}\end{array}$ & $\begin{array}{l}h, e^{\prime}, h^{\prime} \\
a^{\prime}, e^{\prime}, h^{\prime}\end{array}$ \\
\hline
\end{tabular}

This listing is believed to be new, and answers completely the question: What non-redundant triads of undefined functions can define the calculus of sentences?

We know, from the discussion just above, that these triads are non-redun-

(15) Of the fields listed in Table VIII, only 10 were distinct: $F_{i}(i=1,2,3,4,5)$, and the fields for the 5 pairs last investigated. 
dant and we can quickly show that they are sufficient, by the method used in $\S I$ for pairs of functions. We give (as in Table VII for pairs) the definitions, in terms of each triad, of the function $e$, or $b^{\prime}$, either of which can generate $C$.

TABLE XI

\begin{tabular}{lc|}
\hline$a, b, h ;$ & $b^{\prime}=h(b, a)$ \\
$a, h, e^{\prime} ;$ & $e=h\left(e^{\prime}, a\right)$ \\
$b, h, h^{\prime} ;$ & $a=h^{\prime}(f, f) ; b^{\prime}=h(b, a)$ \\
$b, a^{\prime}, h^{\prime} ;$ & $b^{\prime}=h^{\prime}\left(a^{\prime}, b\right)$ \\
$h, e^{\prime}, h^{\prime} ;$ & $a=h^{\prime}(f, f) ; e=h\left(e^{\prime}, a\right)$ \\
$a^{\prime}, e^{\prime}, h^{\prime} ;$ & $e=h^{\prime}\left(a^{\prime}, e^{\prime}\right)$ \\
\hline
\end{tabular}

L. There remain, now, only four non-redundant triads which are still not sufficient. We list them with their fields below:

TABLE XII

$$
\begin{aligned}
& F\left(a, b, a^{\prime}\right)=a, f, g ; b ; a^{\prime} \\
& F\left(a, b, e^{\prime}\right)=a, f, g ; b ; e^{\prime} \\
& F\left(a, a^{\prime}, e^{\prime}\right)=a, f, g ; a^{\prime} ; e^{\prime} \\
& F\left(b, a^{\prime}, e^{\prime}\right)=b, f, g ; a^{\prime} ; e^{\prime}
\end{aligned}
$$

Since $F(\alpha, \beta, \gamma)$ contains at least $f, g, \alpha, \beta, \gamma$, the fields above contain at least the functions listed. To prove the fields closed, consider the following field $F_{6}$.

$$
F_{6}: a, b, f, g, a^{\prime}, e^{\prime}, \text { and the familiar theorem-equations }
$$

$$
\begin{aligned}
a(\beta, \gamma) & =a, & a^{\prime}(\beta, \gamma) & =a^{\prime}, \\
b(a, \beta) & =a, & e^{\prime}(a, \beta) & =\beta, \\
b\left(a^{\prime}, \gamma\right) & =\gamma, & e^{\prime}\left(a^{\prime}, \gamma\right) & =a^{\prime}, \\
b(\beta, \beta) & =\beta, & e^{\prime}(\beta, \beta) & =\beta, \\
b(\beta, \gamma) & =b(\gamma, \beta), & e^{\prime}(\beta, \gamma) & =e^{\prime}(\gamma, \beta) .
\end{aligned}
$$

Since $\beta, \gamma$ are assumed to be in $F_{6}$, the only functions to investigate are those possibly not in $F(b)$ or in $F\left(e^{\prime}\right)$; that is, $b\left(b, e^{\prime}\right)$ and $e^{\prime}\left(b, e^{\prime}\right)$, which turn out to be respectively $b$ and $e^{\prime}$. Obvious applications of these theorems to the triads in Table XII will prove the fields closed as given $\left({ }^{16}\right)$.

Notice that the four non-redundant, insufficient triads are the four triads which can be selected from the tetrad $a, b, a^{\prime}, e^{\prime}$.

(16) These fields are distinct, and are the last fields we shall list. We may indicate here the different fields we have come across in this investigation. The field $C$ is of course generated by every sufficient set. In Table III, there were 11 different fields including $C$. In Table IX, we found 10 different fields, and now just 4 more. We have found, then, 25 fields generated by binary functions, out of the 66 different fields that Post states can be generated by functions of fewer than 4 arguments. 
We have already found complete sets of one, two, and three functions. If a complete set of four undefined functions were to exist, then the condition of non-redundancy demands that the four possible triads which might be selected from it be all non-redundant and insufficient, i.e., that these four possible triads be precisely those of Table XII, that is, if any tetrad is to form a complete set, it must possess at least the properties which are enjoyed only by the tetrad $a, b, a^{\prime}, e^{\prime}$. It must also be capable of defining all functions in $C$. But it is evident from the discussion above that $F\left(a, b, a^{\prime}, e^{\prime}\right)$ is exactly $F_{6}$, that is, $F\left(a, b, a^{\prime}, e^{\prime}\right)=a, b, f, g, a^{\prime}, e^{\prime}$. We have shown, then, that the only non-redundant tetrad is insufficient, and therefore, that there can be no complete tetrad.

To conclude this investigation, we remark that though it is possible to add another function to this tetrad to make it a sufficient quintad, this quintad would be necessarily redundant, since the five possible tetrads in the quintad should all be non-redundant, and there is just one such tetrad: $a, b, a^{\prime}, e^{\prime}$. A fortiori, there exists no complete set of more than five undefined functions.

M. We may summarize our results and translate them into the more familiar language of the calculus of sentences:

1. There are just two complete sets of single functions: $e ; b^{\prime}$; which are Sheffer's stroke function and its dual,- - joint rejection and alternate rejection.

2. There are just 9 complete sets of two functions - those named in Table VI :
negation, implication;
negation, conjunction;
falsity, implication;
verity, non-implication;
negation, non-implication;
negation, disjunction;
non-equivalence, implication;
implication, non-implication.

3. There are just six complete sets of three functions-those named in Table $\mathrm{X}$, or, in translation:
falsity, conjunction, equivalence;
falsity, disjunction, equivalence;
verity, conjunction, non-equivalence;
verity, disjunction, non-equivalence;
conjunction, equivalence, non-equivalence;
disjunction, equivalence, non-equivalence.

4. There is no complete set of more than three functions $\left({ }^{17}\right)$.

NeW York UNIVERSITY,

NEW YoRK, N. Y.

(17) The referee points out that the results are true (in a certain sense) in any Boolean algebra, and not merely in the two-valued calculus of propositions. "That is, the only complete sets of Boolean functions of two variables without parameters are the sets he gives." 